Article

\title{
The Complete Chloroplast Genomes of Two Lancea Species with Comparative Analysis
}

\author{
Xiaofeng Chi ${ }^{1,2,3} \mathbb{B}^{\mathbb{D}}$, Jiuli Wang ${ }^{1,2,3}$, Qingbo Gao ${ }^{1,3}$, Faqi Zhang ${ }^{1,3, *}$ and Shilong Chen ${ }^{1,3, *}$ \\ 1 Key Laboratory of Adaptation and Evolution of Plateau Biota, Northwest Institute of Plateau Biology, \\ Chinese Academy of Sciences, Xining 810001, China; xfchi@nwipb.cas.cn (X.C.); wang_jiul@163.com (J.W.); \\ qbgao@nwipb.cas.cn (Q.G.) \\ 2 University of Chinese Academy of Sciences, Beijing 100049, China \\ 3 Qinghai Provincial Key Laboratory of Crop Molecular Breeding, Xining 810001, China \\ * Correspondence: fqzhang@nwipb.cas.cn (F.Z.); slchen@nwipb.cas.cn (S.C.)
}

Received: 10 February 2018; Accepted: 4 March 2018; Published: 7 March 2018

\begin{abstract}
The genus Lancea is native to the Qinghai-Tibetan Plateau and consists of two species, Lancea tibetica Hook. f. et Thoms. and Lancea hirsuta Bonati. Here, we report the complete sequences of the chloroplast genomes of L. tibetica and L. hirsuta, which were 153,665 and 154,045 bp in length, respectively, and each included a pair of inverted repeated regions (25,624 and 25,838 bp in length, respectively) that were separated by a large single copy region (84,401 and $84,588 \mathrm{bp}$ in length, respectively) and a smaller single copy region (18,016 and 17,781 bp in length, respectively). A total of 106 genes in L. tibetica and 105 in L. hirsuta comprised 79 protein-coding genes, and 4 ribosomal RNA (rRNA) genes, as well as 23 and 22 transfer RNA (tRNA) genes in L. tibetica and L. hirsuta, respectively. The gene order, content, and orientation of the two Lancea chloroplast genomes exhibited high similarity. A large number of informative repetitive sequences, including SSRs, were observed in both genomes. Comparisons of the genomes with those of three other Lamiales species revealed 12 highly divergent regions in the intergenic spacers and in the matK, rpoA, rps19, ndhF, ccs $A, n d h D$, and ycf 1 coding regions. A phylogenomic analysis suggested that Lancea forms a monophyletic group that is closely related to the clade composed of the families Phrymaceae, Paulowniaceae, and Rehmanniaceae.
\end{abstract}

Keywords: Mazaceae; Laminales; organellar genome; phylogenetic analysis

\section{Introduction}

Chloroplasts, which originated from ancient endosymbiotic cyanobacteria, are specialized photosynthetic organelles for photosynthesis and carbon fixation as well as fatty acid synthesis, amino acid synthesis, and the immune response in plants [1]. Chloroplasts also possess their own genomes and genetic systems. Most chloroplast genomes range from 120 to $160 \mathrm{~kb}$ in length and have a typical quadripartite structure with two copies of inverted repeats (IRs) separating the large single copy (LSC) region and the small single copy (SSC) region [2]. Recently, lots of fragments from chloroplast genomes such as $r b c L$ and matK have been widely used in plant systematics research due to its maternal inheritance and highly conserved structures [3]. With the reduction of sequencing cost, the complete chloroplast genome sequences are becoming an increasingly used and effective tool in the study of plant phylogenetic classification, molecular identification and genetic diversity [4]. The comparative analysis of chloroplast genomes is especially useful for inferring new and important insights to resolve many enigmatic phylogenetic relationships for their relatively stable genome structure, gene content, and gene order.

Lancea is a small genus consisting of two species, Lancea tibetica Hook. f. et Thoms. and Lancea hirsuta Bonati, both of which are native to the Qinghai-Tibetan Plateau. The main morphological 
differences between L. tibetica and L. hirsuta are their respective absence and presence of coarse multicellular hairs on their stems and leaves [5]. L. tibetica is found along streams in grasslands and sparse forests at an approximate altitude of 2000-4500 m above sea level in Gansu, Qinghai, Sichuan, and the Tibet region of China as well as Bhutan, India, and Nepal. However L. hirsuta is exclusively endemic to northwest Sichuan, northwest Yunnan, southeast Qinghai and the Tibet region of China [6]. L. tibetica also is an important traditional Tibetan medicine that has been used in the treatment of leukemia, intestinal angina, heart disease, and coughs [7-9].

The systematic position of Lancea has been debated for years. Wettstein (1891) placed Mazus, Dodartia, and Lancea in the tribe Gratioleae, subtribe Mimulinae based on their morphological traits [10]. However, recent molecular studies have indicated that the Scrophulariaceae are not monophyletic [11]. Lancea was then transferred to Phrymaceae according to the phylogenetic analysis of chloroplast $\operatorname{trnL/F}$ and nuclear ribosomal ITS and ETS sequence data [12]. However, no support was found for the monophyly of Phrymaceae. The two subfamilies of Phrymaceae-Phrymoideae and Mazoideae do not form a monophyletic clade in any of the trees in different studies [13]. Consequently, the Angiosperm Phylogeny Group IV (APG IV) classification system separated Lancea, Mazus, and Dodartia from Phrymaceae, placing them into a new family named Mazaceae, which is the outgroup to Paulowniaceae and Orobanchaceae [14]. Although chloroplast and/or nuclear DNA data provide some information about the taxonomy of Lancea, different phylogenetic relationships were inferred when phylogenies were constructed using different sequence fragments, thus requiring confirmation by the use of comprehensive genomic data [11-13,15-17]. Until now, comparative genomics approaches to the study of genetic diversity and phylogenetics in Mazaceae has been limited.

In the present study, we report the complete chloroplast genomes of L. tibetica and L. hirsuta, which were derived using next-generation sequencing. To our knowledge, this is the first report on complete chloroplast genome in the Mazaceae family. Genomic information about L. tibetica and L. hirsuta is fundamental to supporting current conservation efforts especially phylogenetic analysis of these rare species. Our aim was to compare the full chloroplast genomes of these two species, which will serve as valuable genomic resources.

\section{Materials and Methods}

\subsection{DNA Extraction and Sequencing}

L. tibetica was sampled from a single plant collected in Qumalai $\left(96^{\circ} 34^{\prime} 38.8^{\prime \prime} \mathrm{E}, 33^{\circ} 58^{\prime} 03.1^{\prime \prime} \mathrm{N}\right.$, Qinghai, China), while a single sample of $L$. hirsuta was collected from Zaduo $\left(95^{\circ} 00^{\prime} 16^{\prime \prime} \mathrm{E}, 32^{\circ} 51^{\prime} 51^{\prime \prime} \mathrm{N}\right.$, Qinghai, China). The DNA of the two species was isolated from fresh leaves via the modified CTAB method [18]. The DNA content was measured using a NanoDrop spectrophotometer (Thermo Scientific, Carlsbad, CA, USA). Each DNA sample was randomly fragmented to construct paired-end libraries according to the Illumina preparation manual (San Diego, CA, USA). We sequenced the complete chloroplast genomes using the Illumina MiSeq platform at Novogene Biotech Co. (Beijing, China).

\subsection{Chloroplast Genome Assembly and Annotation}

Genomic sequences were screened and assembled with SOAPdenovo [19]. To test the assemble accuracy around IR-LSC/SSC junctions, four primers listed in Table S1 were used to amplify the junctions of IRs and the LSC/SSC. These PCR products were analyzed via Sanger sequencing using the primers mentioned above. Annotation was performed with CpGAVAS (http:/ /www.herbalgenomics. org/cpgavas) [20] coupled with manual adjustment of start/stop codons and intron/exon borders after BLAST searches. The gene homologies were confirmed by comparing them with NCBI's non-redundant $(\mathrm{Nr})$ protein database, Clusters of orthologous groups for eukaryotic complete genomes (KOG), KEGG (http:/ / www.kegg.jp/), GO (http:/ / www.geneontology.org), PFAM (http:/ /xfam.org), SWISS-PROT (http:/ / web.expasy.org/docs/swissprotguideline.html), and TREMBL (http:/ / www. bioinfo.pte.hu/more/TrEMBL.htm) databases. TRNAscan-SE 1.21 were introduced to confirm the 
transfer RNAs (tRNAs). The circular maps of the two Lancea chloroplast genomes were drawn with OrganellarGenomeDRAW (OGDRAW; http:/ / ogdraw.mpimp-golm.mpg.de/index.shtml) [21]. The annotated genomic sequences have been submitted to GenBank under accession numbers MF593117 and MG551489 for L. tibetica and L. hirsuta, respectively.

\subsection{Repeat Structure Analysis}

Dispersed and palindromic repeats were identified by the REPuter program (http:/ / bibiserv2. cebitec.uni-bielefeld.de/reputer) [22]. The minimal size was set to $30 \mathrm{bp}$ with $>90 \%$ identity (Hamming distance equal to 3) between the two repeats. MSDB 2.4 (https:/ / code.google.com/archive/p/msdb / downloads) [23] was used to detect simple sequence repeats (SSRs) with minimal repeat numbers of 10, 5, 4, 3, 3, and 3 for mono-, di-, tri-, tetra-, penta-, and hexa-nucleotides, respectively.

\subsection{Genome Comparison}

The mVISTA program (http://genome.lbl.gov/vista/mvista/about.shtml) [24] was employed in Shuffle-LAGAN mode to determine differences in the chloroplast genomes of L. tibetica and L. hirsuta with those of Rehmannia chingii (KX426347), Paulownia coreana (KP718622), and Erythranthe lutea (KU705476), all of which were obtained from GenBank. The nucleotide variability (average pairwise divergence) between the L. tibetica and L. hirsuta chloroplast genomes was calculated using DnaSP v5.10 (http:/ / www.ub.edu/dnasp/DnaSP_OS.html) [25] with a sliding window analysis. Window length was set to $400 \mathrm{bp}$, and the step size was $200 \mathrm{bp}$.

\subsection{Phylogenetic Analysis}

Phylogenetic analysis was performed among L. tibetica, L. hirsuta, and 21 outgroup Lamiales species (Table S2) on sequence alignments in two ways; one on the complete chloroplast genome sequences, and the other on 75 protein-coding genes. Lactuca sativa (Asteraceae) was used as the outgroup. First, sequences were aligned using MAFFT v7.0 (http://mafft.cbrc.jp/alignment/server/) [26]. Then, jModelTest2 implemented on XSEDE (2.1.6) at the CIPRES Science Gateway (http:/ / www.phylo.org/) was used to select the best model for maximum likelihood (ML) and standard Bayesian inference (BI) analysis. ML analysis was implemented using RAxML-HPC2 on XSEDE (8.2.10) based on the GTR + G + I nucleotide substitution model as recommended by jModelTest 2 with 1000 replications. Similarly, BI analysis was constructed by MrBayes on XSEDE (3.2.6) based on the GTR + G + I nucleotide substitution model. Two independent Markov chain Monte Carlo (MCMC) chains were run for 10,000,000 generations and sampled every 1000 generations with the first $25 \%$ of calculated trees was discarded as burn-in. All the generated trees were modified by Interactive Tree Of Life (iTOL, http:/ /itol.embl.de/) [27].

\section{Results}

\subsection{Characteristics of the Chloroplast Genomes}

The L. tibetica and L. hirsuta chloroplast genomes were 153,655 bp and 154,045 bp in length, respectively. The genomes were like those of most angiosperms with a typical quadripartite structure consisting of a pair of inverted repeats (IRs) of 25,624 bp in L. tibetica and 25,838 bp in L. hirsuta, a large single copy (LSC) region of 84,401 bp in L. tibetica and 84,588 bp in L. hirsuta, and a small single copy (SSC) region of 18,016 bp in L. tibetica and 17,781 bp in L. hirsuta (Figure 1, Table 1). The GC content of the genomes were both $37.9 \%$, but the IR regions had higher GC contents ( $43.3 \%$ and $43.2 \%$ in L. tibetica and L. hirsuta, respectively) than that of the LSC regions (35.9\% and $35.8 \%$ in L. tibetica and L. hirsuta, respectively) and SSC regions (30\% and 32\% in L. tibetica and L. hirsuta, respectively). 


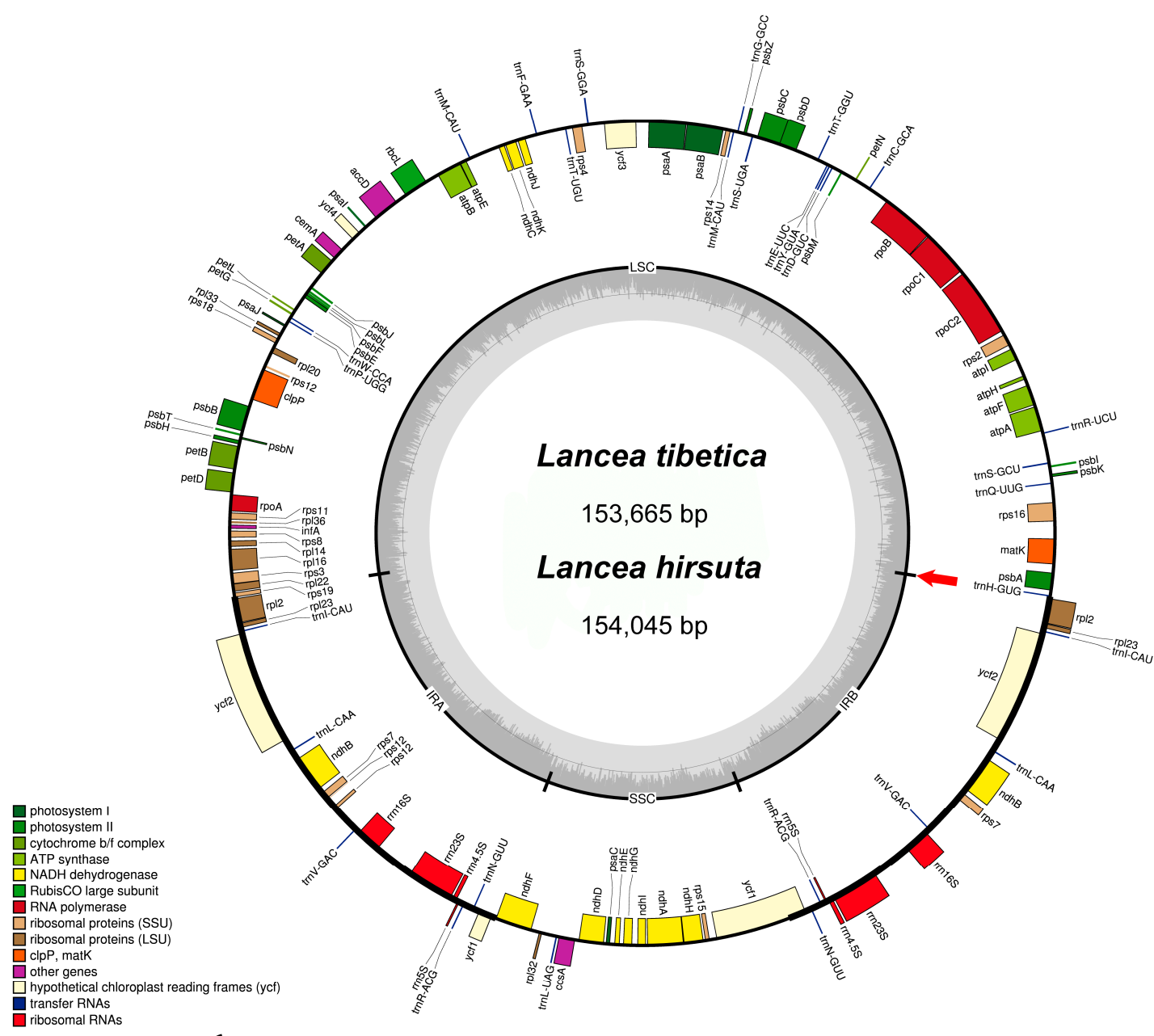

Figure 1. Gene map of the two Lancea chloroplast genomes. Genes belonging to different functional groups are color-coded. Genes drawn inside the circle are transcribed clockwise, while outside are counterclockwise. Nucleotide position 1 was indicated by the red arrow and the sequence was in counterclockwise. Gene trnT-UGU was not found in L. hirsuta.

Table 1. The basic chloroplast genome characteristics of Lancea tibetica and L. hirsuta.

\begin{tabular}{ccc}
\hline Characteristics & L. tibetica & L. hirsuta \\
\hline Total cpDNA size (bp) & 153,664 & 154,045 \\
Length of large single copy (LSC) region & 84,401 & 84,254 \\
Length of inverted repeat (IR) region & 25,624 & 25,838 \\
Length of small single copy (SSC) region & 18,016 & 17,781 \\
Total GC content (\%) & 37.9 & 37.9 \\
LSC & 35.9 & 35.8 \\
IR & 43.3 & 43.2 \\
SSC & 30.0 & 32.0 \\
Total number of genes & 106 & 105 \\
Protein-coding genes & 79 & 79 \\
rRNAs genes & 4 & 4 \\
tRNAs genes & 23 & 22 \\
\hline
\end{tabular}

The gene content, order, and orientation were similar across the two Lancea genomes. There were 106 predicted genes in L. tibetica including 79 protein-coding genes, 23 tRNA genes, and 4 rRNA genes, while the 105 genes predicted in L. hirsuta consisted of 79 protein-coding genes, 22 tRNA genes, and 
4 rRNA genes (Table 2). Among the protein-coding genes, 63 were located in the LSC region, 11 were in the SSC region, and 6 genes $(n d h B, r p l 2, r p l 23, r p s 7$ and $y c f 2)$ were duplicated in the IR regions. There were 13 intron-containing protein-coding genes, two of which (ycf3 and $c l p P$ ) contained two introns. As in most other land plants, the rps12 gene was a trans-spliced gene, with its $5^{\prime}$ end located in the LSC region and its duplicated $3^{\prime}$ ends in the IR regions. The $n d h D$ gene contained the alternative ACG start codon, while rps19 started with GTG, which are common features of most homologous genes in the chloroplast genomes of other plants [28-32]. Approximately 54.8\% of Lancea chloroplast genomes consisted of protein-coding genes (84,254 bp in L. tibetica and 84,474 bp in L. hirsuta), 1.4\% of tRNAs (2198 bp L. tibetica and $2131 \mathrm{bp}$ in L. hirsuta), and 6.1\% of rRNAs (9396 bp in both species). As such, the non-coding regions consisting of introns, pseudogenes, and intergenic spacers accounted for $37.7 \%$ of the both genomes.

Table 2. Genes present in Lancea tibetica and L. hirsuta chloroplast genomes.

\begin{tabular}{|c|c|}
\hline Category & Name \\
\hline Rubisco & $r b c L$ \\
\hline Photosystem I & $p s a A, B, C, I, J$ \\
\hline Photosystem II & $p s b A, B, C, D, E, F, H, I, J, K, L, M, N, T, Z$ \\
\hline ATP synthase & $\operatorname{atp} A, B, E,{ }^{*} F, H, I$ \\
\hline Cytochrome $\mathrm{b} / \mathrm{f}$ complex & $\operatorname{pet} A,{ }^{*} B, * D, G, L, N$ \\
\hline Cytochrome c synthesis & $\operatorname{ccs} A$ \\
\hline NADPH dehydrogenase & ${ }^{*} n d h A,{ }^{*}, \mathrm{a} B, C, D, E, F, G, H, I, J, K$ \\
\hline Transcription & $\operatorname{rpo} A, B,{ }^{*} C 1, C 2$ \\
\hline Small subunit ribosomal proteins & $\operatorname{rps} 2,3,4,{ }^{a} 7,8,11,{ }^{* *} 12,14,15, * 16,18,19$, \\
\hline Large subunit ribosomal proteins & $*$ a $r p l 2,14, * 16,20,22$, a $23,3233,36$ \\
\hline Translation initiation factor & $\inf A$ \\
\hline Ribosomal RNA & a $\operatorname{rrn} 4.5$, a $5,{ }^{\text {a }} 16,{ }^{\text {a }} 23$ \\
\hline RNA processing & matK \\
\hline Carbon metabolism & сетA \\
\hline Fatty acid synthesis & $a c c D$ \\
\hline Proteolysis & ${ }^{* *} c 1 p P$ \\
\hline Unknown function protein-coding gene & $y c f 1,2, * * 3,4$ \\
\hline Transfer RNA & $\begin{array}{l}\operatorname{trnC} C-G C A, \operatorname{trn} D-G U C, \operatorname{trn} E-U U C, \operatorname{trnF}-G A A, \operatorname{trn} G-G C C, \operatorname{trn} H-G U G, \\
\text { a trnI-CAU, a trnL-CAA, trnL-UAG, a trnM-CAU, a trnN-GUU, } \\
\text { trnP-UGG, trnQ-UUG, a trnR-ACG, trnR-UCU, trnS-GCU, trnS-GGA, } \\
\text { trnS-UGA, trnT-GGU, b trnT-UGU, a trnV-GAC, trnW-CCA, trnY-GUA }\end{array}$ \\
\hline
\end{tabular}

${ }^{*}$ gene with one intron, ${ }^{* *}$ gene with two introns, ${ }^{a}$ gene with two copies, ${ }^{b}$ gene not found in L. hirsuta.

\subsection{Repeat and SSR Analysis}

Total of 22 forward repeats and 24 palindromic repeats were found in the L. tibetica genome, while there were 20 forward repeats and 28 palindromic repeats in L. hirsuta (Figure 2A, Tables S3 and S4). The majority of repeats ranged in size from 30 to $44 \mathrm{bp}$, and the longest palindromic repeat (410 bp) was found in L. hirsuta. The repeats were mostly distributed in the intergenic spacers (IGS) and intron sequences, but eight repeats were also found in the coding sequences (CDSs) of $p s a B, y c f 3, r p s 19, n d h B$, $n d h A$ and $y c f 2$.

Simple sequence repeats (SSRs) are another important type of repeated sequence in genomes that are particularly useful molecular markers in genetic diversity research. A total of 50 perfect microsatellites, $574 \mathrm{bp}$ in length, were detected in L. tibetica chloroplast genome, and there were 37, $3,2,4,1$, and 3 mono-, di-, tri-, tetra-, penta- and hexa-nucleotides repeats, respectively (Table S5). In L. hirsuta, the 46 SSRs with totally 496 bp in length included 37, 2, 2, 4, and 1 mono-, di-, tri-, tetra-, and penta-nucleotide repeats, respectively (Table S6). No hexa-nucleotide repeats were found in L. hirsuta. Most SSRs were located in non-coding regions, especially in the LSC region. AT content comprised $86 \%$ and $87 \%$ of SSRs in L. tibetica and L. hirsuta, respectively. 


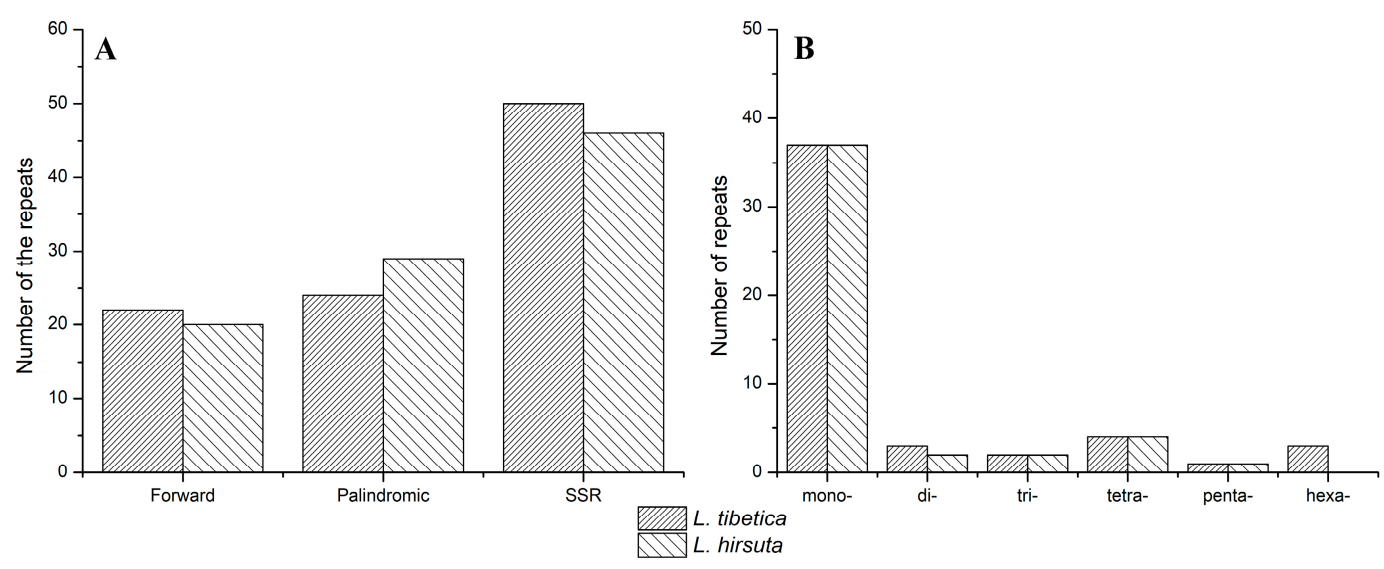

Figure 2. Repeated sequences in Lancea chloroplast genomes. (A) Number of three repeat types within chloroplast genomes; (B) SSR type distribution within Lancea chloroplast genome.

\subsection{IR Contraction and Expansion}

Contraction and expansion at the borders of IR regions have been commonly reported in chloroplast genomes, which may explain the apparent size differences between chloroplast genomes [17]. Accordingly, the inferred assembly was checked to confirm contraction and expansion. Although the IR region of the five chloroplast genomes was highly conserved, structure variation was still found in the IR/SC boundary regions. As shown in Figure 3, the rps19-rp12 gene was located in the junctions of the LSC/IRb regions. The rps19 gene crossed the LSC/IRb region with 3-52 bp located in the IRb region. The ycf1-ndhF gene was located at the junctions of the IRb/SSC regions, though the $t r n N-n d h F$ sequence in E. lutea was missing the $y c f 1$ gene in the IRb region. The $y c f 1$ genes of $R$. chingii, L. tibetica, and L. hirsuta spanned the $\mathrm{IRb}$ and SSC regions, with 3-130 bp in the SSC region. The $n d h F$ gene in P. coreana extended into the LSC region and overlapped with the ycf1 gene by $42 \mathrm{bp}$, while in E. lutea it extended $36 \mathrm{bp}$ into the LSC region. The SSC/IRa junctions in all five chloroplast genomes were crossed by ycf1, with 751-1084 bp in the IRa region. Like the IRb/SSC boundary regions, the LSC/IRa regions were also variable. The $r p l 2-t r n H$ genes of R. chingii, L. tibetica, and L. hirsuta were located at the junctions of IRa/LSC regions with 0, 114, and $106 \mathrm{bp}$, respectively, separating the spacer from the ends of the IRa regions. However, in P. coreana, the rps19 pseudogene was at one end of the IRa region. In E. lutea, the rpl2 gene was missing, and rpl23 was the last gene, with $1607 \mathrm{bp}$ between the spacer and the ends of the IRa regions. Overall, contraction and expansion of the IR regions was detected across the five chloroplast genomes.

\subsection{Sequence Divergence and Divergence Hotspot}

To characterize genome divergence, we performed multiple sequence alignments between the five chloroplast genomes using the program mVISTA, with $R$. chingii being used as a reference (Figure 4). The comparison demonstrated that the coding regions are more conserved than the non-coding regions. In particular, the IR regions were less divergent than the LSC and SSC regions. The most highly divergent regions among the five chloroplast genomes were found among the intergenic spacers, including trnH-psbA, matk-rps16, rps16-psbK petN-psbM, psbZ-rps14, psaA-ycf3, rps4-ndhJ, ndhC-atpE, petA-psbJ, and ycf4-cemA in LSC as well as rpl32-ccs $A$ and $n d h G-n d h I$ in SSC. More divergence of coding regions was found in the $m a t K, r p o A, r p s 19, n d h F, c c s A, n d h D$, and $y c f 1$ sequences. Similar results have been observed in previous studies $[29,32]$. 


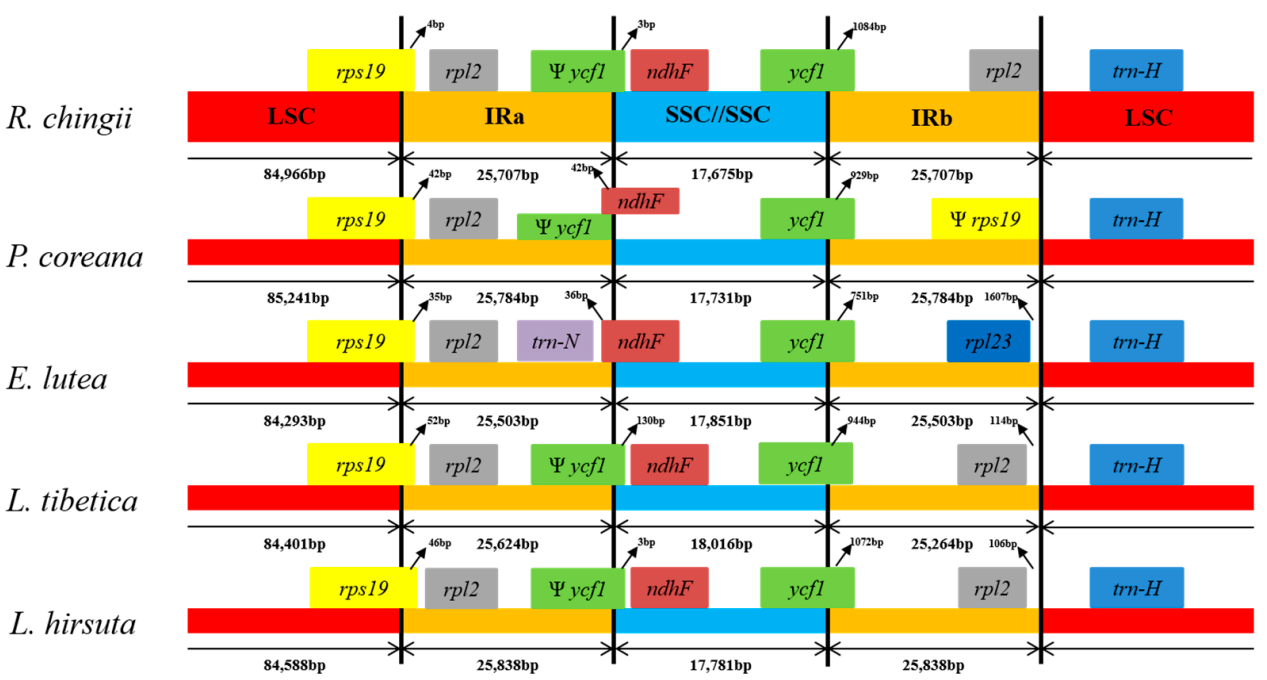

Figure 3. Comparison of the borders of large single-copy (LSC), small single-copy (SSC), and inverted repeat (IR) regions among the chloroplast genomes of five species.

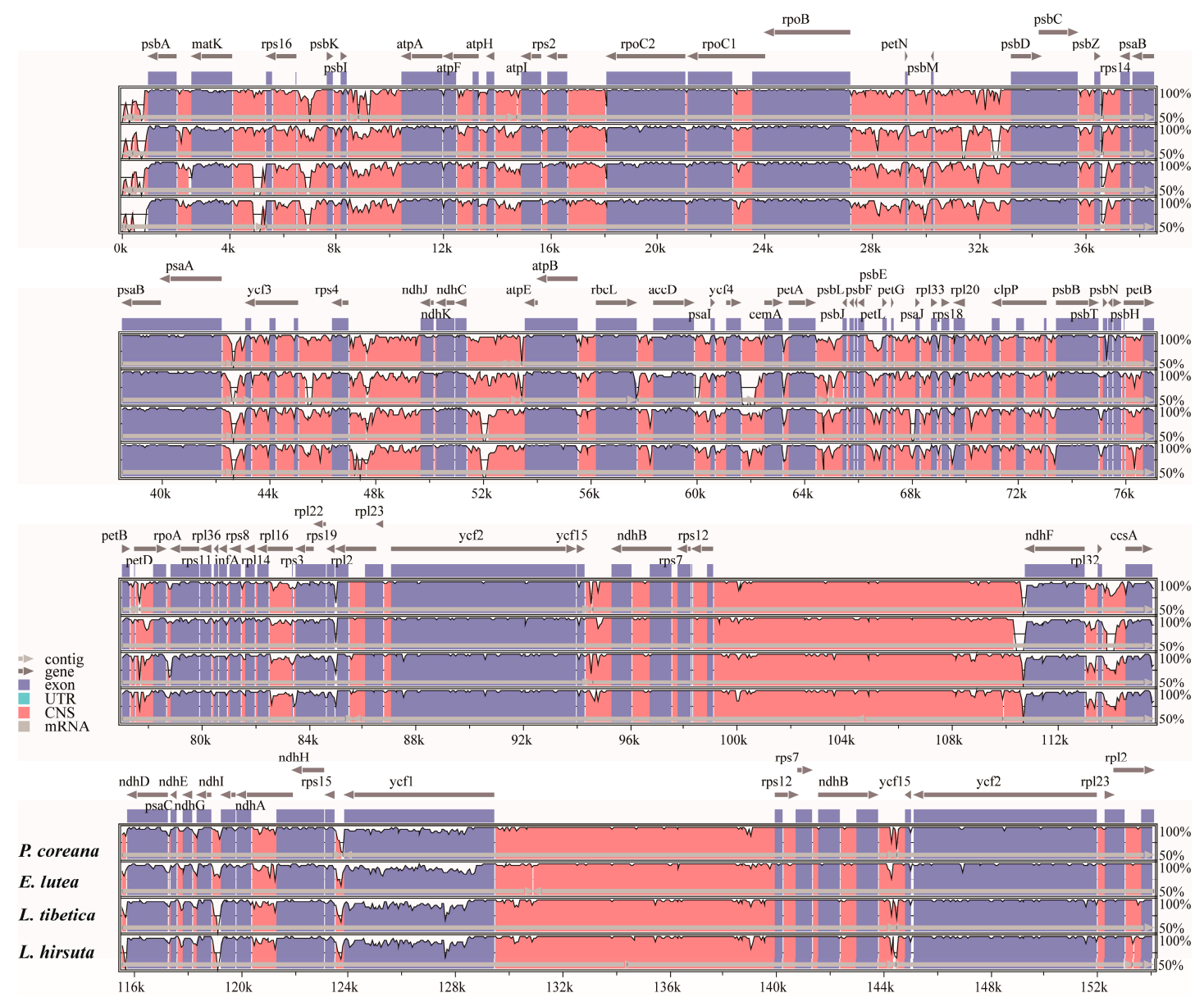

Figure 4. Comparison of five chloroplast genomes using the mVISTA alignment program with Rehmannia chingii as a reference. The $x$-axis represents the coordinates in the chloroplast genome. The $y$-axis indicates the average percent identity of sequence similarity in the aligned regions, ranging between $50 \%$ and $100 \%$. Genome regions are color coded as protein coding, rRNA coding, tRNA coding or conserved noncoding sequences (CNS). 
Nucleotide variability (pairwise divergence) was calculated to show divergence at the sequence level between the two Lancea chloroplast genomes. Between L. tibetica and L. hirsuta, the pairwise divergence values ranged from 0 to 0.09 , with a mean of 0.00221 . As shown in Figure 5 , the IR regions were more conserved than the LSC and SSC regions. The most divergent region, $r p s 4-n d h J$, showed a pairwise divergence value of 0.09 in the LSC region. The petB gene in the LSC region showed the highest degree of nucleotide variability, with a pairwise divergence of 0.0467 . The low divergence values between the L. tibetica and L. hirsuta chloroplast genomes illustrated high similarity between the two species.

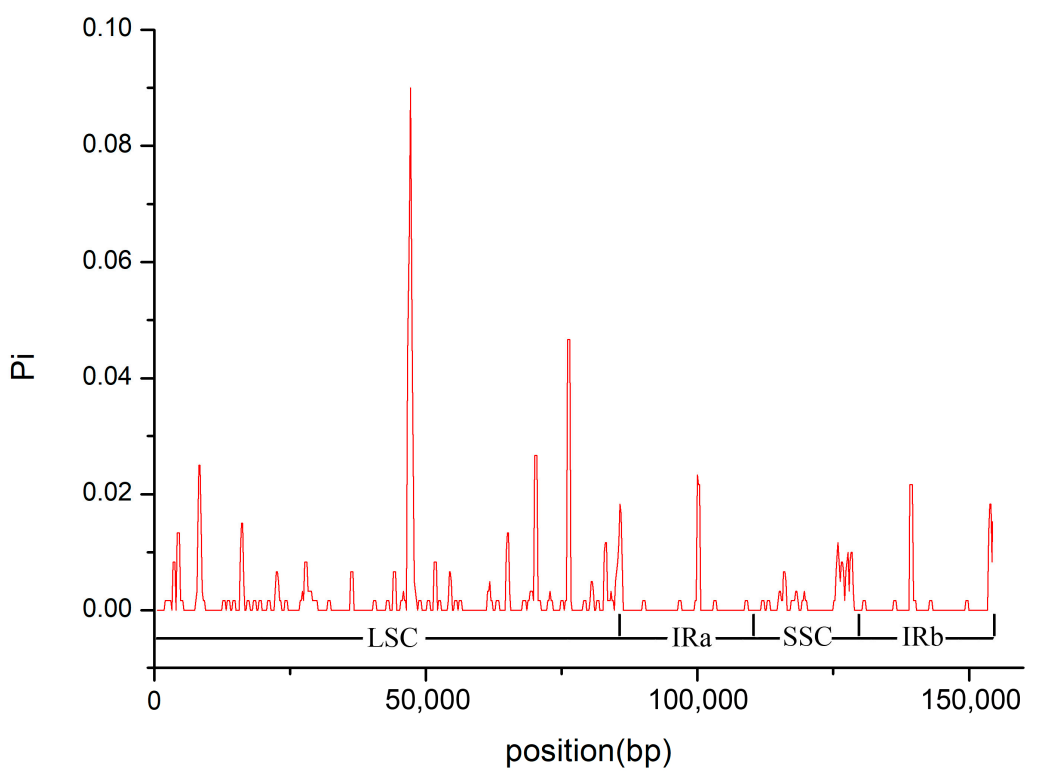

Figure 5. Sliding window analysis of nucleotide variability (pairwise divergence) between Lancea tibetica and L. hirsuta.

\subsection{Phylogenomic Analysis}

Lancea was traditionally placed in Scrophulariaceae. Recent studies have reported its phylogenetic relationship among other genera in the Lamiales based on chloroplast and/or nuclear ribosomal sequence data. However, the position of Lancea was still unclear, and thus required confirmation with additional data. In our present studies, the ML and BI analysis of the complete chloroplast genomes and 75 protein-coding genes showed that the two Lancea species were clustered into one monophyletic group (Figure 6). Alignment of the complete chloroplast genome sequences gave an obvious conflict between ML phylogenetic trees and BI phylogenetic trees, which may be caused by rapidly evolving and potentially poorly aligned sites $[4,33,34]$. On the other hand, alignment of 75 protein-coding genes strongly supported the Lancea genus as sister to a clade formed by Phrymaceae, Paulowniaceae, and Rehmanniaceae, rather than the Scrophulariaceae clade both in ML and BI phylogenetic trees. The relationships supported by our analysis are basically consistent with APG IV [14]. As there is a lack of published chloroplast genomes from the Mazus, Dodartia, and Phrymaceae taxa, the phylogenetic placement of Mazaceae and Phrymaceae remains uncertain. 

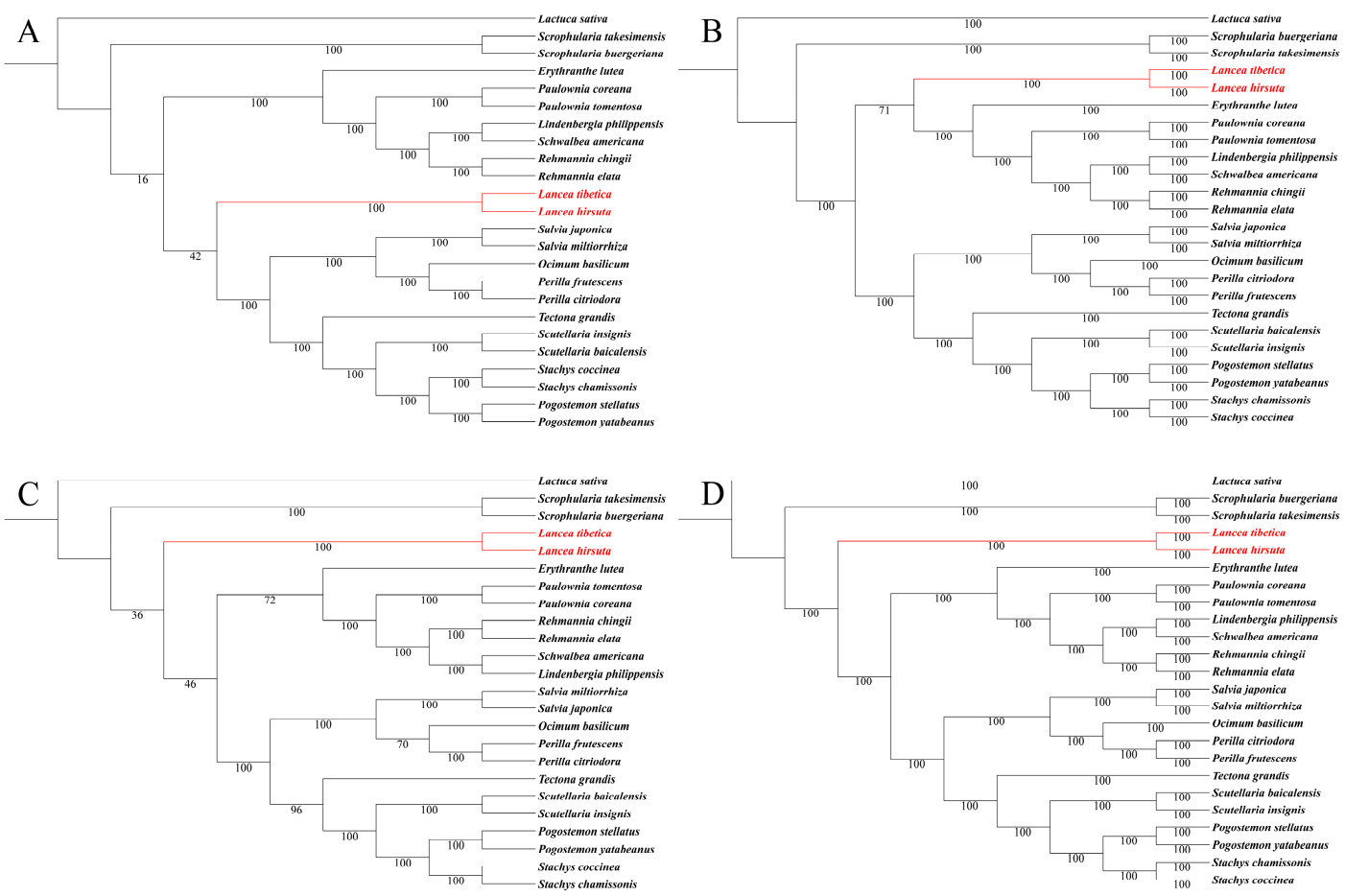

Figure 6. Phylogenetic trees of 24 species based on complete chloroplast genomes and 75 protein-coding genes. (A) Maximum likelihood (ML) phylogenetic tree constructed with complete chloroplast genomes; (B) Bayesian inference (BI) phylogenetic tree constructed with complete chloroplast genomes; (C) ML phylogenetic tree constructed with 75 protein-coding genes; (D) BI phylogenetic tree constructed with 75 protein-coding genes. The Lancea species are shown in red.

\section{Discussion}

Using next-generation sequencing data, two complete Lancea chloroplast genomes were assembled, annotated, and analyzed. In the future, we plan to analyze chloroplast genomes of the genera Mazus and Dodartia, which were placed in Mazaceae in APG IV [14], in order to elucidate the phylogenetic relationships between Lancea and those species. Hence, the comprehensive data presented in this study not only characterizes the entire Lancea chloroplast genomes and enables the inference of their phylogenetic relationships, but also offers a valuable resource for future studies.

Supplementary Materials: Supplementary materials are available online. Table S1: List of all pairs of primers used for assembly validation; Table S2: The list of accession numbers of the chloroplast genome sequences used in the phylogenetic analysis; Table S3: Long repeat sequences in the Lancea tibetica chloroplast genome; Table S4: Long repeat sequences in the Lancea hirsuta chloroplast genome; Table S5: Distribution of SSRs in the Lancea tibetica chloroplast genome; Table S6: Distribution of SSRs in the Lancea hirsuta chloroplast genome.

Acknowledgments: This work was supported by the Applied Basic Research Programs of Qinghai Province (2016-ZJ-761) and Qinghai Provincial Key Laboratory of Crop Molecular Breeding (2017-ZJ-Y14).

Author Contributions: Shilong Chen and Faqi Zhang conceived and designed the research framework; Jiuli Wang prepared the sample and performed the experiments; XiaoFeng Chi analyzed the data and wrote the paper. Qingbo Gao made revisions to the final manuscript. All authors have read and approved the final manuscript.

Conflicts of Interest: The authors declare no conflict of interest.

\section{References}

1. Neuhaus, H.E.; Emes, M.J. Nonphotosynthetic metabolism in plastids. Annu. Rev. Plant Biol. 2000, 51, 111-140. [CrossRef] [PubMed]

2. Sugiura, M. The chloroplast genome. Plant Mol. Biol. 1992, 19, 149-168. [CrossRef] [PubMed] 
3. Daniell, H.; Lin, C.; Yu, M.; Chang, W. Chloroplast genomes: Diversity, evolution, and applications in genetic engineering. Genome Biol. 2016, 17, 134. [CrossRef] [PubMed]

4. Zhang, S.; Jin, J.; Chen, S.; Chase, M.W.; Soltis, D.E.; Li, H.; Yang, J.; Li, D.; Yi, T. Diversification of Rosaceae since the Late Cretaceous based on plastid phylogenomics. New Phytol. 2017, 214, 1355-1367. [CrossRef] [PubMed]

5. Zheng, Y.; Raven, P.H. Flora of China: Vol. 18 Scrophulariaceae through Gesneriaceae. Scrophulariaceae through Gesneriaceae; Missouri Botanical Garden: St. Louis, MO, USA, 1998.

6. Chi, X.; Zhang, F.; Tian, Z.; Chen, S. New records of Lancea hirsuta in Qinghai-Tibetan Plateau. Acta Bot. Boreali-Occident. Sin. 2017, 37, 1447-1449.

7. Song, Z.; Qian, Z.; Rumalla, C.S.; Smillie, T.J.; Khan, I.A. Identification of 11 marker compounds simultaneously in herb Lancea tibetica by using high-performance thin-layer chromatography. JPC 2011, 24, 312-315.

8. Song, Z.; Wang, Y.; Qian, Z.; Smillie, T.J.; Khan, I.A. Quantitative determination of 10 phenylpropanoid and lignan compounds in Lancea tibetica by high-performance liquid chromatography with uv detection. Planta Medica 2011, 77, 1562-1566. [CrossRef] [PubMed]

9. Su, B.; Zhu, Q.; Gao, K.; Yuan, C.; Jia, Z. Lignan and phenylpropanoid glycosides from Lancea tibetica and their antitumor activity. Planta Medica 1999, 65, 558-561. [CrossRef] [PubMed]

10. Wettstein, R. Nolanaceae, Solanaceae, Scrophulariaceae; Engelmann: Leipzig, Germany, 1891.

11. Olmstead, R.G.; Reeves, P.A. Evidence for the polyphyly of the Scrophulariaceae based on chloroplast $r b c L$ and $n d h F$ sequences. Ann. Mo. Bot. Gard. 1995, 82, 176-193. [CrossRef]

12. Beardsley, P.M.; Olmstead, R.G. Redefining Phrymaceae: The placement of Mimulus, tribe Mimuleae, and Phryma. Am. J. Bot. 2002, 89, 1093-1102. [CrossRef] [PubMed]

13. Refulio-Rodriguez, N.F.; Olmstead, R.G. Phylogeny of Lamiidae. Am. J. Bot. 2014, 101, 287-299. [CrossRef] [PubMed]

14. The Angiosperm Phylogeny Group. An update of the angiosperm phylogeny group classification for the orders and families of flowering plants: APG IV. Bot. J. Linn. Soc. 2016, 181, 1-20.

15. Oxelman, B.; Kornhall, P.; Olmstead, R.G.; Bremer, B. Further disintegration of Scrophulariaceae. Taxon 2005, 54, 411-425. [CrossRef]

16. Tank, D.C.; Beardsley, P.M.; Kelchner, S.A.; Olmstead, R.G. Review of the systematics of Scrophulariaceae s.l. and their current disposition. Aust. Syst. Bot. 2006, 19, 289-307. [CrossRef]

17. Xia, Z.; Wang, Y.; Smith, J.F. Familial placement and relations of Rehmannia and Triaenophora (Scrophulariaceae s.1.) inferred from five gene regions. Am. J. Bot. 2009, 96, 519-530. [CrossRef] [PubMed]

18. Doyle, J.J. A rapid DNA isolation procedure for small amounts of fresh leaf tissue. Phytochem. Bull. 1987, 19, 11-15.

19. Luo, R.; Liu, B.; Xie, Y.; Li, Z.; Huang, W.; Yuan, J.; He, G.; Chen, Y.; Qi, P.; Liu, Y. SOAPdenovo2: An empirically improved memory-efficient short-read de novo assembler. Gigascience 2012, 1, 18. [CrossRef] [PubMed]

20. Liu, C.; Shi, L.; Zhu, Y.; Chen, H.; Zhang, J.; Lin, X.; Guan, X. CpGAVAS, an integrated web server for the annotation, visualization, analysis, and genbank submission of completely sequenced chloroplast genome sequences. BMC Genom. 2012, 13, 715. [CrossRef] [PubMed]

21. Lohse, M.; Drechsel, O.; Bock, R. Organellargenomedraw (OGDRAW): A tool for the easy generation of high-quality custom graphical maps of plastid and mitochondrial genomes. Curr. Genet. 2007, 52, 267-274. [CrossRef] [PubMed]

22. Kurtz, S.; Choudhuri, J.V.; Ohlebusch, E.; Schleiermacher, C.; Stoye, J.; Giegerich, R. REPuter: The manifold applications of repeat analysis on a genomic scale. Nucleic Acids Res. 2001, 29, 4633-4642. [CrossRef] [PubMed]

23. Du, L.; Li, Y.; Zhang, X.; Yue, B. MSDB: A user-friendly program for reporting distribution and building databases of microsatellites from genome sequences. J. Hered. 2012, 104, 154-157. [CrossRef] [PubMed]

24. Frazer, K.A.; Pachter, L.; Poliakov, A.; Rubin, E.M.; Dubchak, I. VISTA: Computational tools for comparative genomics. Nucleic Acids Res. 2004, 32, W273-W279. [CrossRef] [PubMed]

25. Librado, P.; Rozas, J. DnaSP v5: A software for comprehensive analysis of DNA polymorphism data. Bioinformatics 2009, 25, 1451-1452. [CrossRef] [PubMed]

26. Kazutaka, K.; Standley, D.M. MAFFT multiple sequence alignment software version 7: Improvements in performance and usability. Mol. Biol. Evol. 2013, 30, 772-780. 
27. Letunic, I.; Bork, P. Interactive Tree Of Life (iTOL) v3: An online tool for the display and annotation of phylogenetic and other trees. Nucleic Acids Res. 2016, 44, W242-W245. [CrossRef] [PubMed]

28. Yi, D.K.; Kim, K.J. Two complete chloroplast genome sequences of genus Paulownia (Paulowniaceae): Paulownia coreana and P. tomentosa. Mitochondrial DNA Part B 2016, 1, 627-629. [CrossRef]

29. Park, I.; Kim, W.J.; Yeo, S.M.; Choi, G.; Kang, Y.M.; Piao, R.; Moon, B.C. The complete chloroplast genome sequences of Fritillaria ussuriensis maxim. and Fritillaria cirrhosa D. Don, and comparative analysis with other Fritillaria species. Molecules 2017, 22, e982. [CrossRef] [PubMed]

30. Shen, X.; Wu, M.; Liao, B.; Liu, Z.; Bai, R.; Xiao, S.; Li, X.; Zhang, B.; Xu, J.; Chen, S. Complete chloroplast genome sequence and phylogenetic analysis of the medicinal plant Artemisia annua. Molecules 2017, 22, e1330. [CrossRef] [PubMed]

31. Wu, M.; Li, Q.; Hu, Z.; Li, X.; Chen, S. The complete Amomum kravanh chloroplast genome sequence and phylogenetic analysis of the commelinids. Molecules 2017, 22, 1875. [CrossRef] [PubMed]

32. Zeng, S.; Zhou, T.; Han, K.; Yang, Y.; Zhao, J.; Liu, Z.L. The complete chloroplast genome sequences of six Rehmannia species. Genes 2017, 8, 103. [CrossRef] [PubMed]

33. Zhong, B.; Deusch, O.; Goremykin, V.V.; Penny, D.; Biggs, P.J.; Atherton, R.A.; Nikiforova, S.V.; Lockhart, P.J. Systematic error in seed plant phylogenomics. Genome Biol. Evol. 2011, 3, 1340-1348. [CrossRef] [PubMed]

34. Som, A. Causes, consequences and solutions of phylogenetic incongruence. Brief. Bioinform. 2015, 16, 536-548. [CrossRef] [PubMed]

Sample Availability: Sequence data of two Lancea species are available from the authors.

(C) 2018 by the authors. Licensee MDPI, Basel, Switzerland. This article is an open access article distributed under the terms and conditions of the Creative Commons Attribution (CC BY) license (http://creativecommons.org/licenses/by/4.0/). 